\title{
Is vaccine hesitancy justified? Benchmarking post-market vaccine risks with five commonly used medicinal products in Canada
}

\author{
Tuhin Maity ${ }^{1}$ (I) $\cdot$ Ahmad von Schlegell ${ }^{1,2} \cdot$ Christopher Longo $^{1}$ \\ Received: 3 May 2021 / Accepted: 6 January 2022 / Published online: 11 February 2022 \\ (C) The Author(s) under exclusive license to The Canadian Public Health Association 2022
}

\begin{abstract}
Objective Although vaccines are one of the most cost-effective, low-risk healthcare approaches that save thousands of lives every year, paradoxical fear about vaccine safety is a major roadblock for achieving widespread vaccination coverage. The objective of this study is to change public perception of vaccine safety by presenting real-world incidence of adverse events following immunization (AEFIs).

Methods In this study, we used Canadian post-market adverse events data to estimate the real-world risk of AEFI and benchmarked them against five commonly used drug types-ACE inhibitors, beta2 adrenergic receptors, penicillins, proton pump inhibitors, and HMG-CoA reductase inhibitors.

Results Our analysis shows that post-market AEFIs are rare, and vaccination generally carries a significantly lower risk compared to some commonly used medicinal product types.

Conclusion Despite some limitations with using post-trial adverse events data, we believe that the evidence presented in this study, especially the comparative risk analysis between vaccines and medicinal products, when communicated through proper channels, can help vaccine-hesitant individuals overcome their perceived safety concerns with regard to vaccines.
\end{abstract}

\section{Résumé}

Objectifs Bien que les vaccins soient l'une des approches de soins de santé les plus rentables et à faible risque qui sauvent des milliers de vies chaque année, la peur paradoxale de la sécurité des vaccins est un obstacle majeur à la réalisation d'une couverture vaccinale généralisée. L'objectif de cette étude est de changer la perspective publique de la sécurité des vaccins en présentant l'incidence actuelle des événements indésirables post-commercialisation après la vaccination.

Méthodes Dans cette étude, nous avons utilisé les données canadiennes sur les événements indésirables post-commercialisation pour estimer le risque réel d'événements indésirables après la vaccination et les avons comparés à cinq types de médicaments couramment utilisés - inhibiteurs de l'ECA (enzyme de conversion de l'angiotensine), récepteur bêta-2-adrénergique, pénicillines, inhibiteurs de la pompe à protons et inhibiteurs de l'HMG-CoA réductase.

Résultats Notre analyse montre que les événements indésirables post-commercialisation après la vaccination sont rares et que la vaccination comporte généralement un risque significativement plus faible par rapport à certains types de médicaments couramment utilisés.

Conclusion Malgré certaines limites à l'utilisation des données sur les événements indésirables post-essai, nous pensons que les preuves présentées dans cette étude, en particulier l'analyse comparative des risques entre les vaccins et les médicaments, lorsqu'elle est communiquée par des canaux appropriés, peuvent aider les personnes hésitantes à surmonter leurs préoccupations perçues en matière d'innocuité des vaccins.

Tuhin Maity

maityt@mcmaster.ca

1 Health Policy and Management, DeGroote School of Business,

McMaster University, 1280 Main Street West,

Hamilton, Ontario L8S 4M4, Canada

2 Credit Valley Hospital, Trillium Health Partners,

Mississauga, Ontario, Canada 
Keywords Vaccine $\cdot$ Vaccination refusal $\cdot$ Adverse effects $\cdot$ Side effects $\cdot$ Adverse drug reaction reporting systems

Mots-clés Vaccin · refus de vaccination · effets indésirables · effets secondaires · systèmes de déclaration des effets indésirables des médicaments

\section{Introduction}

After access to affordable nutrition, clean drinking water, and sanitation, vaccines are the single most cost-effective healthcare measure that can be taken to protect human health (Morrow et al., 2012). All vaccines approved in Canada (and in most developed countries) undergo a thorough safety and efficacy evaluation based on the data from the clinical trials (Health Canada, 2020). In fact, since vaccines are usually administered to healthy individuals, they are typically held to a higher standard of safety than the medicinal products used to treat ill patients (Destefano et al., 2018).

Nonetheless, despite the success of vaccines, they have never been free from the paradoxical concerns about their safety (Chen, 1999; Larson et al., 2011). Although adverse events following immunization (AEFIs) are rare, a perceived risk of AEFIs plays a central role in instigating vaccine hesitancy among individuals, thereby acting as a barrier in achieving widespread vaccination coverage (Larson et al., 2016). According to the World Health Organization, vaccine hesitancy is defined as delay in acceptance or refusal of vaccines despite availability of vaccine services (MacDonald, 2015). Vaccine hesitancy is typically influenced by factors such as complacency, convenience, and confidence (MacDonald, 2015). In a recent survey in Canada about COVID-19 vaccines, the top two reasons for vaccine hesitancy among individuals were found to be lack of confidence in the safety of vaccines $(54.2 \%)$ and concerns about their risks and side effects (51.7\%) (Frank \& Arim, 2020). About one third of Canadians (34.8\%) who are unlikely to get vaccinated indicated that they would wait until enough data suggest that it is safe to get the vaccine (Frank \& Arim, 2020).

Boosting public confidence in the benefits of vaccination by underscoring the rarity of AEFIs is a critically required component for achieving widespread vaccination coverage (Goldstein et al., 2015). However, even large clinical trials are not necessarily always powered to detect rare AEFIs (Jacobson et al., 2001). In addition, clinical trials generally confine attention to idealized standard patients and are conducted in idealized conditions to determine efficacy. Enhanced post-market surveillance offers real-world evidence for decision making by individuals, providers, policy makers, and society (Duclos, 2004).

The overarching objective of this study is to change public perception of vaccine safety by presenting real-world incidence of AEFIs. Reports from the Canada Vigilance
Adverse Reactions (CVAR) database (Canada Vigilance Adverse Reaction Online Database, 2021) were used as the source of post-market adverse incidents of vaccines and medicinal products, and their outcomes. Using the reports from this database, this study presents an overview of AEFIs and their outcomes, and a side-by-side comparison of post-market adverse events (AEs) associated with vaccines and common medicinal products.

\section{Methods}

The CVAR database contains information about suspected adverse reactions to health products, which include both vaccines and medicinal products (Canada Vigilance Adverse Reaction Online Database, 2021). The database includes data from 1965, although information about vaccines used for immunization has been included in the database since 2011 . Health professionals submit AE reports to this database voluntarily. However, market authorization holders, i.e., manufacturers and distributors, are required to submit $\mathrm{AE}$ reports to this database according to the Food and Drug Regulations (Food and Drug Regulations (C.R.C., c. 870), 2021).

Adverse event reports were extracted from the CVAR database for the period 2015-2019. A detailed description of data extraction and processing method can be found elsewhere (Maity \& Longo, 2020; Maity \& Longo, 2021). This study refers to an AEFI or AE as serious based on Health Canada's definition. Health Canada defines an AE as 'serious' if it requires in-patient hospitalization or prolongation of existing hospitalization, causes congenital malformation, results in persistent or significant disability or incapacity, is life-threatening, or results in death (Health Canada, 2019). When an adverse reaction is determined as 'serious', a report to Health Canada must be submitted in an expedited fashion, such as within 15 days by the market authorization holder or within 30 calendar days by the hospital (Food and Drug Regulations (C.R.C., c. 870), 2021). For AEFI outcomes, death, life-threatening conditions, disability, and hospitalization required were considered in this study.

Five vaccine types were chosen to further illustrate the AEFI incidence rates: seasonal influenza, meningococcus, MMR, pneumonia, and shingles (only Zostavax, see below). The selection of these five vaccine types was based on their higher occurrence in the CVAR database and the fact that their 
uptake is widespread among Canadians. Of note, in 2017, Shingrix vaccine received a market authorization in Canada for shingles and began replacing Zostavax. Since Zostavax is a live attenuated vaccine and Shingrix is a recombinant zoster vaccine, we did not combine the data for two different kinds of vaccines for the comparative purpose. Only AEFI data associated with Zostavax were used for shingles. The number of vaccine doses distributed for the five vaccine types was calculated from averaging the number of Ontario doses for 20152018 reported by Public Health Ontario (Annual Report on Vaccine Safety, 2021) after adjusting for the Canadian population using the population information from Statistics Canada (Population Estimates on July 1st, by Age and Sex, 2021). AEFI incidence rate per dose was calculated by dividing the annual average number of incidents - all AEs, serious AEs, and death - by the annual average of doses calculated as described above. Since, for most vaccines, it is just one dose per unique recipient in a given year, the risk per dose can be approximated as risk per recipient.

For benchmarking the AEFI rate with medicinal products, five product classes were selected based on the number of users in Canada (Canadian Institute for Health Information, 2019). They were ACE inhibitors, beta2 adrenergic receptors, penicillins, proton pump inhibitors, and HMG-CoA reductase inhibitors. The use of the CVAR database as a source of AE reports for both vaccines and medicinal products helps reduce some reporting-related biases, such as the extent of underreporting, that may influence the benchmarking. The number of users for these five medicinal product types was obtained from annual prescribed drug spending reports published by the Canadian Institute for Health Information (CIHI) (Canadian Institute for Health Information, 2019). Note, the recipient numbers from 2017 to 2019 were used to calculate the average, since the data for 2015 and 2016 were not available in the same data series from CIHI.

Raw data for AEFIs and AEs associated with the five vaccines and five medicinal product types, respectively, will be available upon request to the corresponding author.

\section{Results}

\section{AEFIs in Canada}

A total of 4083 AEFI reports were identified for vaccines in the CVAR database for the period 2015-2019. Table 1 presents an overview of the reports and their outcomes. On average, there were 817 reports per year, with 108 patient hospitalizations, 19 life-threatening conditions and 12 deaths from the suspected AEFIs (Table 1). The number of AEFI reports in the CVAR database more than doubled from 2015 to 2019 . This increase was not a vaccine-specific phenomenon. As illustrated in Fig. 1, the number of $\mathrm{AE}$ reports for other medicinal products excluding vaccines in the database also doubled from 2015 to 2019 (Fig. 1). For vaccines, the proportion of serious AEFIs increased from 58\% in 2015 to $74 \%$ in 2019 (Fig. 1A). For medicinal products excluding vaccines, the proportion of serious AEs remained quite constant around $72 \%$.

\section{Vaccine types and AEFls}

Table 2 presents the top vaccine types that are associated with the 4083 AEFI reports. One or multiple vaccines may be associated with each AEFI report. When only one vaccine is associated, that vaccine is automatically considered as a suspect for causing the AEFI. When more than one vaccine is associated, at least one vaccine is named as suspect, and the others as concomitant. Importantly, a classification of suspect does not automatically establish a causal relationship between a vaccine and the adverse reactions. Nevertheless, a suspect/concomitant classification performed by trained health practitioners implies the degree of likelihood of a causal relationship.

\section{Benchmarking AEFI rates}

To portray the risk or rarity of AEFIs, we converted the AEFI incidents into events per number of recipients or doses and then benchmarked them against the AE rates observed for some commonly used medicinal products estimated from the same database.
Table 1 AEFIs associated with vaccines and their outcomes from the CVAR database

\begin{tabular}{llllll}
\hline Year & All reports & Death & Disability & $\begin{array}{l}\text { Life- } \\
\text { threatening }\end{array}$ & Hospitalization required \\
\hline 2015 & 563 & 7 & 37 & 11 & 75 \\
2016 & 524 & 9 & 29 & 12 & 68 \\
2017 & 557 & 8 & 28 & 25 & 67 \\
2018 & 1061 & 15 & 39 & 25 & 146 \\
2019 & 1378 & 19 & 68 & 23 & 186 \\
Total & 4083 & 58 & 201 & 96 & 542 \\
Annual average & 817 & 12 & 40 & 19 & 108 \\
\hline
\end{tabular}


A

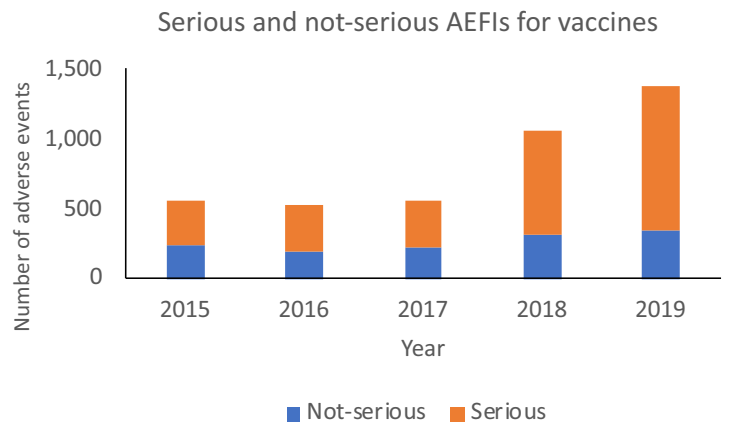

B

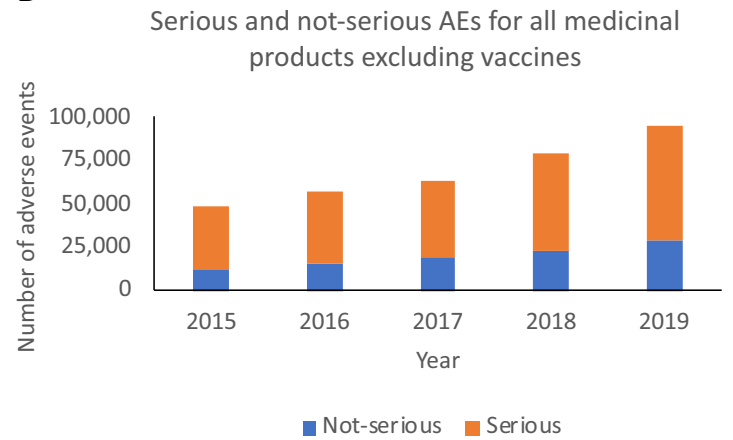

Fig. 1 Serious and not-serious AEs for vaccines and all medicinal products excluding vaccines. The numbers of annual reports in the database are presented to illustrate the increase in the number of reports in 2015-2019

Table 3 presents the estimated all $\mathrm{AE}$, serious $\mathrm{AE}$, and attributed fatality rates associated with five vaccines and five medicinal product types. For four of the five vaccine types, fewer than 10 AEFIs and serious AEFIs were associated with every 100,000 doses. Attributed fatality from the AEFIs was extremely rare, typically in the order of 1 or 2 per million doses.

The medicinal product types analyzed in this study exhibited a relatively higher rate of AEs, serious AEs, and AE-associated deaths compared to the five vaccine types (Table 3). Among the drugs, penicillins appeared to exhibit the lowest rate of AEs per 100,000 users.

\section{Interpretation, future direction, and limitations}

Overall, our benchmarking analysis shows that post-market AEFIs are rare and vaccination generally carries a

Table 2 Vaccine types in the AEs in 2015-2019

\begin{tabular}{llll}
\hline Vaccine type & Concomitant & Suspect & All \\
\hline BCG & 35 & 26 & 61 \\
D/T/P & 115 & 244 & 359 \\
Influenza & 379 & 804 & 1183 \\
Hep** & 88 & 234 & 322 \\
Meningococcus & 21 & 87 & 108 \\
MMR & 15 & 123 & 138 \\
Others & 35 & 176 & 211 \\
Pneumonia & 299 & 551 & 850 \\
Shingles (Zostavax and Shingrix) & 100 & 1628 & 1728 \\
Varicella & 83 & 107 & 190 \\
Total & $\mathbf{1 1 7 0}$ & $\mathbf{3 9 8 0}$ & $\mathbf{5 1 5 0}$ \\
\hline
\end{tabular}

*The total is more than 4083 as some reports contain more than one vaccine

**Hep includes Hepatitis A, Hepatitis B, and the combined Hepatitis A and $B$ vaccines significantly lower risk even when compared with some commonly used medicinal product types.

We observed that the total number of AEFIs gradually increased from 2015 to 2019 (Table 1 and Figure 1A). This is unlikely due to an overall increase in the risk of AEFIs or the rate of vaccination. Pharmaceutical products excluding vaccines exhibited a very similar trend (Fig. 1B). Over this period, the number of $\mathrm{AE}$ reports per million population changed substantially for all medicinal products (Maity \& Longo, 2020). This suggests that an increased rate of reporting is likely the primary driver for the observed increase in the number of AEFI reports in the database. An increased number of reports may also be due to increases in vaccine uptake due to increased eligibility or promotion of vaccines, as well as new vaccines (e.g., Zostavax), in addition to increased awareness about reporting to CVAR.

For both medicinal products and vaccines, the majority of the AEs reported in the database are serious in nature (Figure 1). Unlike in clinical trials, where AEs of all seriousness levels are documented, a post-market database containing spontaneously collected reports is likely to be biased towards serious events. Mild events that do not require medical intervention are more likely to be underreported.

The association between a vaccine or medicinal product to an $\mathrm{AE}$ in the CVAR database does not imply causality. An assessment of causality requires consideration of other factors, such as temporal associations, the possible contribution of concomitant medication or therapies, and the underlying health condition of the patient. The assessment of suspect vs. concomitant classification in the CVAR database reflects the observation and opinion of the reporter (Table 2). Though an investigation and assessment of causality is not formally carried out for reports in CVAR, when a vaccine or medicinal product is considered a suspect in an AE, it is more likely to reflect a causal connection compared to when it is considered concomitant.

Among the vaccines in the benchmarking analysis, Zostavax exhibited a higher rate of AEFIs compared to the other vaccines. Zostavax is a live attenuated vaccine and the 
Table 3 Benchmarking the average AEFI rate for five vaccines against the average AE rate for the top five medicinal products using reports from the CVAR database for the period 2015-2019

Events per $100 \mathrm{~K}$ recipients/users*

Vaccines

Type (doses)

Influenza $(10,659,843)$

Meningococcus $(964,137)$

MMR $(786,059)$

Pneumonia $(1,857,669)$

Shingles (Zostavax) (321,578)

$\begin{array}{lll}\text { All AEs } & \text { Serious AEs } & \text { Dea } \\ 2.2 & 1.5 & 0.1 \\ 2.2 & 1.8 & 0.0 \\ 3.5 & 2.8 & 0.1 \\ 9.2 & 6.6 & 0.2 \\ 34.5 & 22.8 & 0.6\end{array}$

Medicinal products

\begin{tabular}{llll}
\hline Type (users) & All AEs & Serious AEs & Deaths \\
\hline ACE inhibitors $(1,825,482)$ & 145.5 & 114.7 & 9.5 \\
Beta2 adrenergic receptors $(1,776,150)$ & 231.0 & 198.9 & 16.0 \\
Penicillins $(2,110,266)$ & 41.8 & 32.7 & 2.3 \\
Proton pump inhibitors $(2,653,363)$ & 273.5 & 218.6 & 16.8 \\
HMG-CoA reductase inhibitors $(3,589,419)$ & 122.2 & 95.8 & 9.3 \\
\hline
\end{tabular}

*Assuming that for most vaccines there is a 1:1 ratio between their number of doses and the number of unique recipients. Annual average numbers of doses for vaccines and users for medicines are provided in the parentheses. For vaccines, the annual average was calculated with only doses distributed in the publicly funded programs in Ontario and then extrapolated for Canada, and, therefore, is underestimated. This overestimates the AEFI rates for vaccines.

recipients are exclusively older adults. It is not totally unexpected to observe a relatively higher AEFI rate associated with a live vaccine. Nevertheless, the estimated rates of all AEs, serious AEs, and attributed fatalities associated with any of the five vaccines were lower than those of the five medicinal products investigated in this study. Note, these medicinal products are not representative of all medicinal products in terms of their safety. We speculate that they are likely perceived as safer prescribed medicines as they are routinely and widely used without much hesitancy (Hales \& Kohen, 2019).

In a larger context, the question is whether this benchmarking data on its own is sufficient to create a shift in the vaccine-hesitant individual's mindset. Strong evidence of vaccine safety derived from real-world data such as that presented in this study may not automatically convert vaccine-hesitant individuals - whose hesitancy is caused by the fear associated with vaccines' unintended side effectsinto vaccine recipients.

Vaccine-hesitant individuals are more likely to distrust experts (e.g., scientists, healthcare professionals) and authority (e.g., the state), and hold conspiratorial and paranoid beliefs (Murphy et al., 2021). Vaccine-hesitant individuals are less likely to consume and trust information from traditional sources, such as news articles, radio, television, and government agencies (Murphy et al., 2021). The comparative safety information presented in this study needs to be put into context and the delivery of this information must be tailored to overcome the barriers described above. We believe that disseminating vaccine safety information such as that presented in this paper, and effective communication through alternative and unconventional channels by individuals who the vaccine-hesitant population trust the most, are likely to reduce vaccine hesitancy.

Analysis using spontaneously collected post-market reports has some limitations. First, underreporting is a known issue in post-market $\mathrm{AE}$ data, which leads to underestimation of the risk (Hazell \& Shakir, 2006). However, we mitigated this concern for the relative risk comparison by using the same database for AEs associated with vaccines and medicinal products. Second, we sourced the vaccine user information from Health Quality Ontario, which only included users in the publicly funded programs. Therefore, the actual numbers of recipients for the five vaccine types considered here are likely higher, leading to a systematic overestimation of the AEFI rates not seen in the medicinal product arm. Furthermore, while there could be inter-provincial variations, by extrapolating the vaccine uptake rate for Ontario to Canada, we have tacitly assumed that Ontario vaccine uptake is generally representative of the entire nation. This is not an unreasonable assumption. As of September 18, 2021, 75.25\% of Ontarians received at least one dose of COVID-19 vaccine (COVID-19 Vaccination in Canada, 2021). For the same time period, $75.26 \%$ Canadians have received at least one dose, a number that is almost identical to that of Ontario (COVID-19 Vaccination in Canada, 2021). Third, while for most vaccines there is a 1:1 ratio between their number of doses and the number of unique recipients, the medicinal products included in benchmarking are typically prescribed to use in a continuous manner over a period or lifetime, and the number of doses administered is typically much higher than the number of users. Therefore, a question may arise as to whether our approach led to a fair benchmarking analysis. We argue that the AE rates estimated in this study practically reflect the number of events annually per 100,000 users. Furthermore, for medicinal products, the risk is ongoing due to their use in a continuous manner over a period of time. For vaccines, the risk is not ongoing for an individual, as the number of doses for most vaccines is typically limited by the number of doses in a specified time period, 


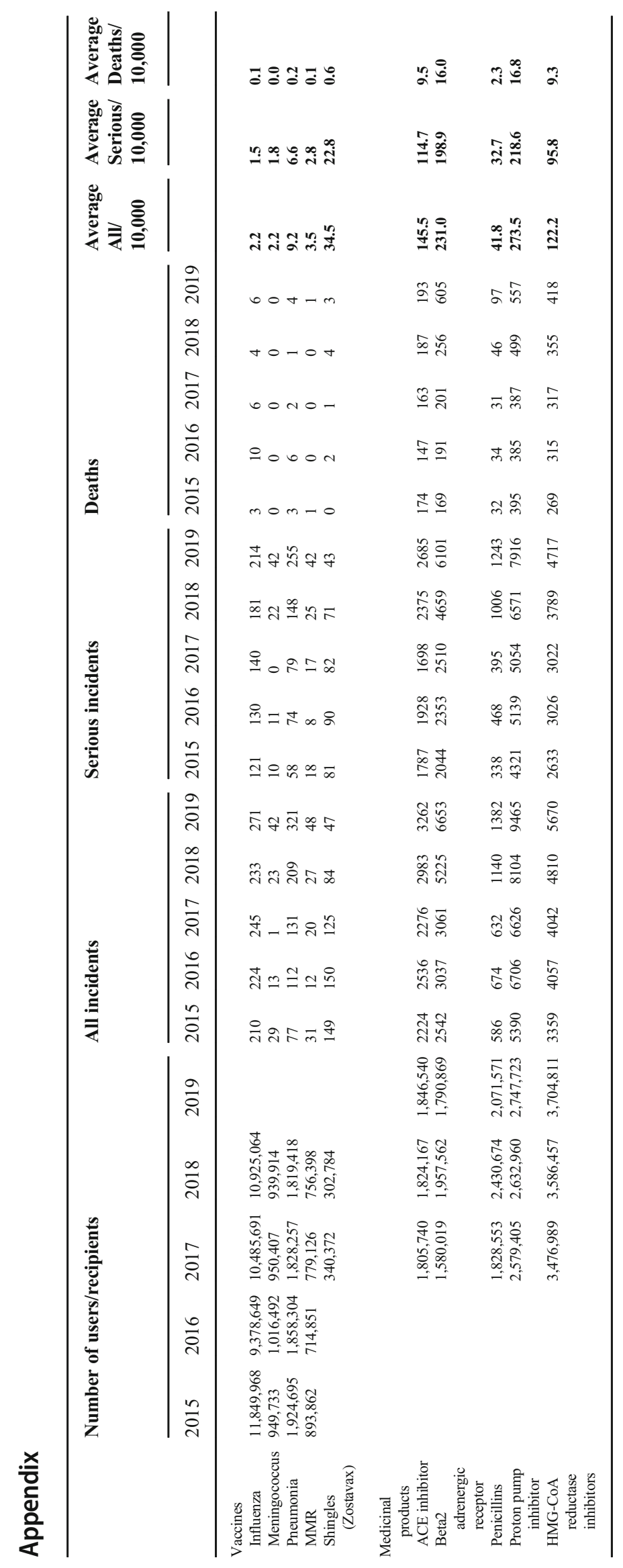


ranging from annually for influenza to longer intervals or even once in a lifetime for others. Nevertheless, we expect that some researchers may want to use a different approach for benchmarking. Fourth, an association between a vaccine or medicinal product and an AE does not automatically indicate causality. When multiple vaccines or medicinal products are involved, it is even harder to establish a causal connection. In addition, patient co-morbidities also play a significant role in AE outcomes. Since medicinal products are generally used by sick individuals, AEs associated with medicinal products may tend to be more frequent compared to the vaccines, which are generally administered to healthy individuals. Therefore, comparing AEFIs and AEs associated with medicinal products without controlling for co-morbidities may lead to a favourable bias towards vaccines, although it is highly unlikely this would account for a 100 -fold difference in frequency.

\section{Conclusion}

Perceived fear of AEFIs is a major hurdle for achieving widespread vaccination coverage, needed for herd immunity. In this study, we used the CVAR database, a repository of post-market AEs in Canada, to estimate the real-world rates of AEFIs. Our results show that AEFIs are rare and vaccination generally carries a significantly lower risk even when compared with some commonly used medicinal product types. We hope that the results of our study, when communicated through proper channels, will improve our understanding and perception of vaccine safety in the real world and improve vaccine uptake.

\section{Contributions to knowledge}

What does this study add to existing knowledge?

- This study benchmarks adverse event rates for vaccines and medicinal products using spontaneously collected Canadian post-market adverse events data.

- This study shows that adverse events following immunization are rare, and vaccination generally carries a significantly lower risk compared to some commonly used medicinal product types.

What are the key implications for public health interventions, practice or policy?

- The evidence presented in this study, when communicated through proper channels, should help vaccine-hesitant individuals overcome their perceived safety concerns with regard to vaccines.
Author contributions TM, AV, and CL conceptualized the study. TM extracted and analyzed the data. TM, AV, and CL contributed to writing the manuscript.

Availability of data and material Raw data for adverse events associated with vaccines and the five drug types in this study will be available upon request to the corresponding author.

Code availability Not applicable

\section{Declarations}

Ethics approval Not applicable

Consent to participate Not applicable

Consent for publication Not applicable

Conflict of interest The authors declare no competing interests.

\section{References}

Annual Report on Vaccine Safety. (2021). [Public Health Ontario]. Vaccine Safety Surveillance Archive. https://www. publichealthontario.ca/en/data-and-analysis/infectious-disease/ vaccine-safety/vaccine-safety-surveillance-archive. Accessed 2021$10-24$.

Canada Vigilance adverse reaction online database. (2021). https://www. canada.ca/en/health-canada/services/drugs-health-products/ medeffect-canada/adverse-reaction-database.html. Accessed 202112-01.

Canadian Institute for Health Information. (2019). Prescribed Drug Spending in Canada, 2019: A Focus on Public Drug Programs. https://www.cihi.ca/en/prescribed-drug-spending-in-canada-2019. Accessed 2021-10-24.

Chen, R. T. (1999). Vaccine risks: Real, perceived and unknown. Vaccine, 17, S41-S46. https://doi.org/10.1016/s0264-410x(99) 00292-3

COVID-19 vaccination in Canada. (2021). https://health-infobase. canada.ca/covid-19/vaccination-coverage/. Accessed 2021-10-17.

Destefano, F., Offit, P. A., \& Fisher, A. (2018). Vaccine safety. Plotkin's Vaccines, 1584-1600.e10. PMC. https://doi.org/10.1016/B978-0323-35761-6.00082-1

Duclos, P. (2004). A global perspective on vaccine safety. Vaccine, 22(15-16), 2059-2063. https://doi.org/10.1016/j.vaccine.2004.01. 010

Food and Drug Regulations (C.R.C., c. 870). (2021). Justice Laws Website. https://laws-lois.justice.gc.ca/PDF/C.R.C.,_c._870.pdf. Accessed 2021-01-03.

Frank, K., \& Arim, R. (2020). Canadians' willingness to get a COVID-19 vaccine: Group differences and reasons for vaccine hesitancy. Statistics Canada. https://www150.statcan.gc.ca/n1/en/pub/45-280001/2020001/article/00073-eng.pdf?st=HwQvZdw3. Accessed 2021-04-15.

Goldstein, S., MacDonald, N. E., \& Guirguis, S. (2015). Health communication and vaccine hesitancy. Vaccine, 33(34), 4212-4214. https:// doi.org/10.1016/j.vaccine.2015.04.042

Hales, C. M., \& Kohen, D. (2019). Prescription drug use among adults aged 40-79 in the United States and Canada (No. 347; NCHS Data Brief, p. 8). U.S. DEPARTMENT OF HEALTH AND HUMAN 
SERVICES. https://pubmed.ncbi.nlm.nih.gov/31442200/. Accessed 2021-10-17.

Hazell, L., \& Shakir, S. A. W. (2006). Under-reporting of adverse drug reactions. Drug Safety, 29(5), 385-396. https://doi.org/10.2165/ 00002018-200629050-00003

Health Canada. (2019). Mandatory reporting of serious adverse drug reactions and medical device incidents by hospitals. [Guidance Document]. https://www.canada.ca/en/health-canada/services/ drugs-health-products/medeffect-canada/adverse-reactionreporting/mandatory-hospital-reporting/drugs-devices/guidance. html. Accessed 2021-10-24.

Health Canada. (2020). Regulating vaccines for human use in Canada. https://www.canada.ca/en/health-canada/services/drugs-healthproducts/biologics-radiopharmaceuticals-genetic-therapies/ activities/fact-sheets/regulation-vaccines-human-canada.html. Accessed 2021-10-17.

Jacobson, R. M., Adegbenro, A., Pankratz, V. S., \& Poland, G. A. (2001). Adverse events and vaccination-the lack of power and predictability of infrequent events in pre-licensure study. Vaccine, 19(17-19), 2428-2433. https://doi.org/10.1016/S0264-410X(00)00467-9

Larson, H. J., Cooper, L. Z., Eskola, J., Katz, S. L., \& Ratzan, S. (2011). Addressing the vaccine confidence gap. The Lancet, 378(9790), 526-535. https://doi.org/10.1016/S0140-6736(11)60678-8

Larson, H. J., de Figueiredo, A., Xiahong, Z., Schulz, W. S., Verger, P., Johnston, I. G., Cook, A. R., \& Jones, N. S. (2016). The state of vaccine confidence 2016: Global insights through a 67-country survey. EBioMedicine, 12, 295-301. https://doi.org/10.1016/j.ebiom. 2016.08 .042
MacDonald, N. E. (2015). Vaccine hesitancy: Definition, scope and determinants. Vaccine, 33(34), 4161-4164. https://doi.org/10.1016/j. vaccine.2015.04.036

Maity, T. S., \& Longo, C. J. (2020). Adverse drug reactions in Canada (2009-2018): Insights from the Canada Vigilance Database. Healthcare Quarterly, 23(1), 40-46. https://doi.org/10.12927/hcq. 2020.26140

Maity, T., \& Longo, C. (2021). Pragmatic pharmacoeconomic analyses by using post-market adverse drug reaction reports: An illustration using infliximab, adalimumab, and the Canada vigilance adverse reaction database. BMC Health Services Research, 21(1), 1231. https://doi.org/10.1186/s12913-021-07260-Z

Morrow, J., Seikh, N., Schmidt, C., \& Davies, H. (2012). Vaccinology: Principles and practice. Wiley.

Murphy, J., Vallières, F., Bentall, R. P., Shevlin, M., McBride, O., Hartman, T. K., McKay, R., Bennett, K., Mason, L., GibsonMiller, J., Levita, L., Martinez, A. P., Stocks, T. V. A., Karatzias, T., \& Hyland, P. (2021). Psychological characteristics associated with COVID-19 vaccine hesitancy and resistance in Ireland and the United Kingdom. Nature Communications, 12(1), 29. https:// doi.org/10.1038/s41467-020-20226-9

Population estimates on July 1st, by age and sex. (2021). Statistics Canada. https://www150.statcan.gc.ca/t1/tbl1/en/tv.action?pid= 1710000501. Accessed 2021-09-29.

Publisher's note Springer Nature remains neutral with regard to jurisdictional claims in published maps and institutional affiliations. 